\title{
Comment on the Prune Belly Syndrome: An 11-Week Fetus With Megacystis
}

\author{
MASON BARR, JR., AND ALPHONSE R. BURDI \\ Departments of Pediatrics (M.B., Jr.) and Anatomy (A.R.B.), University of \\ Michigan, Ann Arbor, Michigan 48109
}

\begin{abstract}
An 11-week human fetus with megacystis, prostatic dysgenesis, and lateral displacement of the abdominal muscles is described. We suggest that a subtle outflow obstruction of the very early bladder may give rise to both bladder dilation and bladder wall dysgenesis. The bladder dilation may produce abdominal muscle dysplasia or atrophy and almost certainly produces dilation of the prostatic urethra. The dilation of the prostatic urethra disrupts the formation of the prostate.
\end{abstract}

Apropos of recent communications on the prune belly syndrome (PBS) (Monie and Monie, '79a, b; Marshall, '79) we would like to describe a fetus that has some bearing on this intriguing condition.

A 66-mm CR, 81-day-old fertilization age male fetus was recovered from a tubal pregnancy in 1947. No other details of its provenance are known. Serial sections, stained with $\mathrm{H}$ $\& \mathrm{E}$, were made of various body regions and stored in The University of Michigan Embryo Collection. Findings of remark are: enlarged kidneys with polyectatic nephrons, pyelocaliectasis, megacystis, medial thinning and lateral displacement of the abdominal musculature, and a single umbilical artery.

The bladder is hugely distended and occupies a major portion of the abdominal cavity (Fig. 1). The distention extends into the prostatic urethra where the prostatic buds are widely dispersed over the external urethral surface. The urethra distal to this point appears to have a normal caliber, and no point of obstruction can be identified. The ureters, at least those portions adjacent to the bladder, are of normal caliber and no ureterovesical obstruction is evident. The midportions of the ureters are not present in the collection and there is no description of their condition. The pelves and calyces of the kidneys are dilated, and there is ectasia of many but not all of the nephrons. The dilation involves entire nephrons from the glomerulus to the distal convoluted tubules, but the maximal dilation is found in the proximal convoluted tubules. The testes lie ventrolateral to the bladder and appear normal in all respects.
The anterior abdominal wall is distended, but each of the abdominal muscles can be identified. Wax plate reconstructions of the rectus abdominis muscles were made from serial saggital sections of the abnormal fetus and a similarly sectioned normal fetus matched for age, size, and sex (Fig. 2). In the normal fetus the rectus is found in its normal elliptical form in cross section. In the abnormal fetus, the main rectus bulk is displaced far laterally, while the medial portion is very attenuated and the interrectus distance is markedly increased. The wax sections were melted down and the volumes of wax compared. The total rectus volumes of the normal and abnormal fetuses are almost identical.

The histology of the skeletal muscle fibers is appropriate for the gestational age. The bladder wall is thin but the appearance of the smooth muscle cells and the porportion between muscle and fibrous tissue are unremarkable. Ganglion cells are present in both the bladder and colon.

That this fetus represents an early example of PBS is arguable. Certainly the configuration of the dilated bladder/prostatic urethra is the same as had been described repeatedly in PBS. However, dilation of the bladder and prostatic urethra has been reported in the presence of normal abdominal musculature (King, '69; Williams and Taylor, '69). The apparent absence of hydroureter argues against PBS, but hydroureter could be a later development, after intravesical pressures had produced decompensation of the

\footnotetext{
Received September 29, 1980; accepted October 29, 1980.
} 


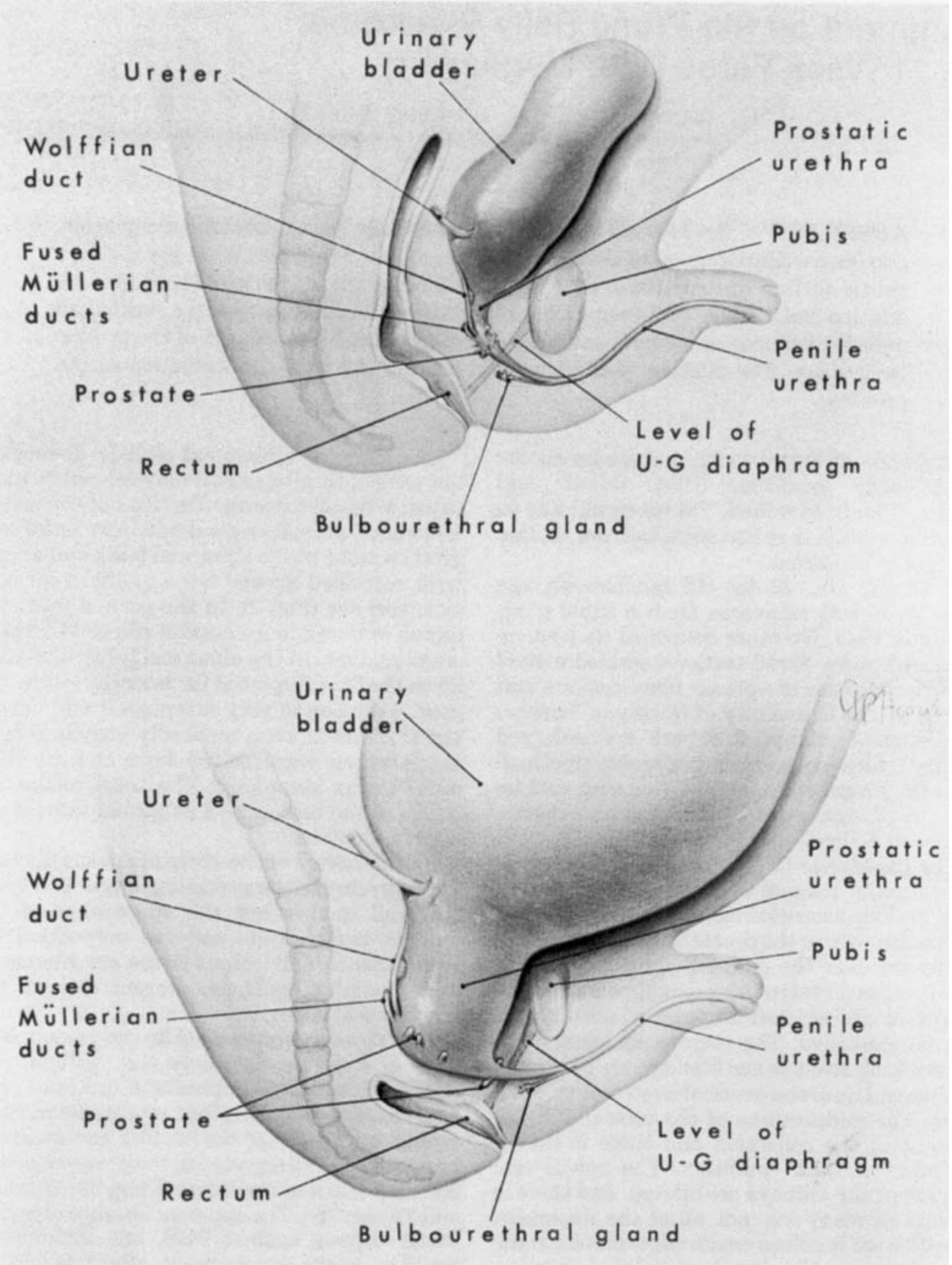

Fig. 1. Fetal pelvic anatomy. Top: Eleven-week $165 \cdot \mathrm{mm}$ CR) fetus with normal pelvic anatomy. Bottom: Eleven- week (66-mm-Ch) fetus with megacystis and dilated prostatic urethra. 
A.

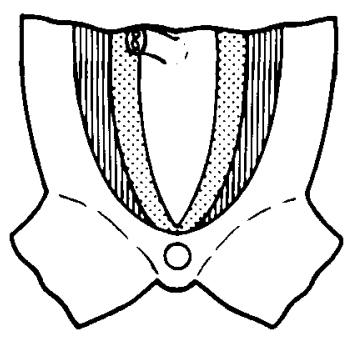

B.

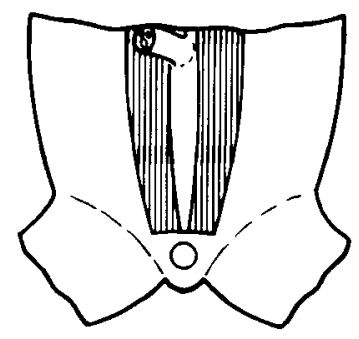

\section{main bulk of rectus muscle}

\section{attenuated rectus muscle}

Fig. 2. Abdominal muscle disposition. A) Eleven-week (66-mm CR) fetus with lateral displacement and medial thinning of the rectus abdominis muscles. B) Eleven-week (65-mm CR) fetus with normal positioning and configuration of the rectus abdominis muscles.

ureterovesical valvular mechanism and vesicoureteral reflux.

At first inspection it would appear that the distended bladder exerted an effect on the abdominal muscles but only by displacing the rectus laterally to produce a diastasis recti. The question is: did the medial borders of the recti fail to reach the midline or were they later displaced toward the sides?

Examination of other specimens in the UM Embryo Collection shows that the definitive position of the rectus abdominis muscles and their sheaths is present by $35 \mathrm{~mm}$ CR $(8.5 \mathrm{wk}$ FA), or well before the age of our abnormal fetus. The bladder originally lies within the abdominal wall. In 25-30 mm-CR embryos the bladder lumen begins to enlarge and push the pos- terior bladder wall out into the peritoneal cavity. This bladder dilation presumably coincides with the onset of significant urine production by the metanephros. Bladder distention and midventral apposition of the rectus muscles are then almost simultaneous events. This confounds the cause/effect question of whether the enlarged bladder affects the abdominal wall or an abdominal wall laxity allows overex. pansion of the bladder.

To be sure, we cannot know what secondary changes might have occurred with subsequent growth. It is not inconceivable that the medial thinning of the rectus abdominis could gradually extend laterally to involve the entire rectus and beyond into the abdominal obliques. However, the assumption that abdominal wall distention produces a secondary atrophy of the muscle cannot be proven or disproven from this case.

Another instructive element of this case is the light it sheds on the proposal of Monie and Monie ('79a) that the initiating factor in PBS may be prostatic dysgenesis. The prostatic buds first appear at 10-11 weeks FA and through subsequent growth coalesce to form the definitive gland. In our fetus, at the early stages of prostatic budding, the urethra is already widely dilated and the buds are widely separated. This convinces us that prostatic dysgenesis is a secondary phenomenon: because of the preexisting urethral dilation, the prostatic buds cannot coalesce into a normal gland.

We argue that bladder distention can have but two causes: 1) high intravesical pressure due to (a) outflow obstruction or (b) high volume load; or 2) normal intravesical pressure in the face of an unresisting bladder wall.

The notion of an obstructive uropathy is an appealing explanation of the megacystis and the nephropyelocaliectasis. Because our sections are inadequate to either rule in or out a ureteropelvic obstruction, no comment on the genesis of the upper urinary tract dilation is justified. However, we can demonstrate that the distal urethra, although much narrower than the prostatic urethra, has a normal caliber and form when compared to an age-matched control. Application of the laws of hydrodynamics supports the notion of a relative obstruction of outflow from the dilated to the normal caliber urethra. But this fails to explain how the dilated portion got that large in the first place. In the absence of demonstrable obstruction, we must consider the possibility that the early bladder/prostatic urethra was 
unusally distensible. This implies a mesenchymal/muscular defect of the early bladder, arising at some point between $11 \mathrm{~mm} C R$ (cloaca divided) and $26 \mathrm{~mm} \mathrm{CR}$ (first two layers of bladder muscle evident) when the bladder has begun to be pushed out of the abdominal wall.

When we consider that PBS occurs predominantly, but not exclusively, in males, this explanation seems to be deficient. It becomes tempting to suggest that the configuration of the male uretha is sufficiently different from the female urethra at $25 \mathrm{~mm}$ CR so that a small and perhaps inapparent restriction in bore could cause enough outflow impedance to initiate an overdistention of the bladder. In that case, the overdistention could interfere with the normal development of bladder musculature with the result of defective wall resistance to further expansion. The distended bladder would then not only balloon out into the peritoneal cavity but also distend the urethra in a proximodistal direction until the intravesical pressure was balanced or exceeded by extravesical (tissue) pressure around the distal urethra.

\section{ACKNOWLEDGMENTS}

We express our gratitude to Gerald Hodge for the drawings of the fetal bladders, to Alayne Spencer-Evans for the drawings of the fetal muscles, and to Penny Romlein for typing the manuscript.

\section{LITERATURE CITED}

King, L.R. (1969) Idiopathic dilation of the posterior urethra in boys without bladder outlet obstruction. J. Urol., 102: 783-787.

Marshall, F.F. (1979) Comments on the prune belly syndrome: letter to the editor. Teratology, 20: 333.

Monie, I.W. and B.J. Monie (1979a) Prune belly syndrome and fetal ascites. Teratology, 19:111-118.

Monie, I.W. and B.J. Monie (1979b) Comments on the prune belly syndrome: author's response. Teratology, 20: 334 .

Williams, D.I. and J.S. Taylor (1969) A rare congenital uropathy: vesico-urethral dysfunction with upper tract anomalies. Br. J. Urol., 41: 307-313. 